\title{
Qualitative chirality effects on the Casimir-Lifshitz torque with liquid crystals
}

\author{
Wijnand Broer $\odot^{*}$ \\ School of Physical Sciences, University of Chinese Academy of Sciences, Beijing 100049, China \\ Bing-Sui Lu $\odot$ \\ Division of Physics and Applied Physics, Nanyang Technological University, Singapore 637371, Singapore \\ Rudolf Podgornik $\oplus^{\dagger}$ \\ School of Physical Sciences, University of Chinese Academy of Sciences, Beijing 100049, China \\ Institute of Physics, Chinese Academy of Sciences, 100190 Beijing, China \\ Kavli Institute for Theoretical Sciences, University of Chinese Academy of Sciences, Beijing 100049, China \\ Wenzhou Institute of the University of Chinese Academy of Sciences, Wenzhou, Zhejiang 325000, China \\ and Department of Theoretical Physics, J. Stefan Institute, 1000 Ljubljana, Slovenia and Department of Physics, \\ Faculty of Mathematics and Physics, University of Ljubljana, 1000 Ljubljana, Slovenia
}

(Received 29 June 2021; accepted 27 August 2021; published 10 September 2021)

\begin{abstract}
We model a cholesteric liquid crystal as a planar birefringent multilayer system, where the orientation of each layer differs slightly from that of the adjacent one. This allows us to analytically simplify the otherwise acutely complicated calculation of the Casimir-Lifshitz torque. Numerical results differ appreciably from the case of nematic liquid crystals, which can be treated like bloc birefringent media. In particular, we find that the torque deviates considerably from its usual sinusoidal behavior as a function of the misalignment angle. In the case of a birefringent crystal faced with a cholesteric liquid one, the Casimir-Lifshitz torque decreases more slowly as a function of distance than in the nematic case. In the case of two cholesteric liquid crystals, either in the homochiral or in the heterochiral configuration, the angular dependence changes qualitatively as a function of distance. In all considered cases, finite pitch length effects are most pronounced at distances of about $10 \mathrm{~nm}$.
\end{abstract}

DOI: 10.1103/PhysRevResearch.3.033238

\section{INTRODUCTION}

Casimir-Lifshitz interactions [1,2] are macroscopic dispersion forces that arise from quantum mechanical and thermal fluctuations of the electromagnetic field. The name "dispersion forces" originates from the fact that their properties are governed by the dielectric and magnetic dispersion of the interacting materials [3-6]. Casimir-Lifshitz forces share the same physical origin as the van der Waals-London forces, which conventionally denote the interaction in cases where retardation effects are negligible.

These forces are studied for both fundamental and practical reasons. From a fundamental point of view, the CasimirLifshitz force plays an important role in the search for deviations from Newtonian gravitation in short-range gravitation experiments. Its study can facilitate the precise comparisons between theoretical predictions and experimental data [7]. More practically, Casimir-Lifshitz interactions

\footnotetext{
*wbroer@gmail.com

†podgornikrudolf@ucas.ac.cn

Published by the American Physical Society under the terms of the Creative Commons Attribution 4.0 International license. Further distribution of this work must maintain attribution to the author(s) and the published article's title, journal citation, and DOI.
}

affect the actuation dynamics of nano and micromechanical systems, such as switches, cantilevers, and actuators at a submicrometer lengthscale [8-13]. The Casimir-Lifshitz interactions also underpin the stability of colloidal and biophysical macromolecular systems. These forces represent one of the pillars of the fundamental Deryaguin-Landau-VerweyOverbeek theory of colloid stability [14].

Precise evaluation of the Casimir-Lifshitz force between real materials requires a detailed knowledge of the materials' electromagnetic susceptibilities as inputs [2]. Because this interaction is mediated by virtual photons, the frequency of which cannot be controlled directly, the Casimir-Lifshitz interaction is a broadband phenomenon. Consequently, the electromagnetic susceptibilities of the materials involved must be known - either theoretically or experimentally — as a function of frequency in a sufficiently broad range. Another requirement to evaluate the Casimir-Lifshitz interaction is solving Maxwell's equations for the given geometric configuration of the interacting bodies. This later aspect of the Casimir-Lifshitz calculations is the focus of this paper.

The presence of anisotropies, either in the susceptibilities or the morphological anisotropy induced by the shape of the interacting bodies [15], implies the existence of not only forces but also Casimir-Lifshitz torques between the interacting bodies [16]. Specifically, the dielectric anisotropy in the plane of reflection, which will exclusively concern us in what follows, creates a dielectric contrast in the 
azimuthal direction, which gives rise to the Casimir-Lifshitz torque attempting to align the orientations of both materials [17]. An exact analytical description of the Casimir-Lifshitz torque between two planar uniaxially anisotropic half spaces was originally based on the direct solution of the Maxwell equations and the pertaining dispersion equation by Barash more than four decades ago [18]. An alternative methodology applicable to the Casimir-Lifshitz torque can be based on the solutions of the Maxwell's equations by interpreting them as an eigenvalue problem. This method is known as the transfer matrix method. It is especially suited for describing electromagnetic field propagation through anisotropic materials [19-21], and can be used to derive the Casimir torque between semi-infinite birefringent plates [22,23]. The results of the transfer matrix methodology were later shown to be consistent with the direct method of Barash [24]. Some recent examples of the application of this method in the context of Casimir physics include biaxial materials [25], Weyl semimetals [26,27], and magnetic ferrite slabs [28].

The first experimental observation of Casimir-Lifshitz torques was accomplished only recently, by relying on the anisotropic dielectric response of nematic liquid crystals [29]. It seems that further experiments on Casimir-Lifshitz torques with liquid crystals are within experimental reach presently or in the near future. Apart from the Casimir-Lifshitz torques, liquid crystals exhibit diverse other Casimir fluctuation phenomena and so might prove to be an abundant source of a variety of distinct Casimir phenomena [30,31]. The simplest way to theoretically describe the anisotropy of liquid crystals is by approximating them as uniaxial half spaces with a unique orientation [32]. This essentially approximates the liquid crystal as a birefringent half space. In what follows we will refer to this approximation as "half space approximation." This is indeed a valid approach if the liquid crystal is aligned, such as in the case of nematic liquid crystals [33]. However, a cholesteric liquid crystal actually consists of a sequence of many thin, planar, uniaxially oriented layers, whose orientation varies periodically in space with a periodicity given by the cholesteric pitch [34]. This type of layered system has been shown to be amenable to the transfer matrix method [19] as electromagnetic waves in layered anisotropic media can be described by an algebra of $4 \times 4$ matrices [35].

However, a major complication arises from the fact that the cholesteric liquid crystal consists of a large number of layers $\left(\sim 10^{4}\right)$, which generally implies a product of an equally large number of $4 \times 4$ matrices. Even though the nonretarded limit reduces the size of the matrices to $2 \times 2$, it does not reduce the number of matrix multiplications [33,36-38]. Since the computational complexity of matrix multiplication increases linearly with the number of multiplications, an explicit analytical expression for the transfer matrix can reduce this complexity by up to four orders of magnitude. Hence here we propose a way to analytically simplify this problem and derive an explicit expression for the composite transfer matrix for a specific planar multilayer structure that models a cholesteric liquid crystal. We have taken advantage of the facts that each layer is very thin, and that the difference in orientation between each successive layer is small [34]. This allows us to write an analytic result for the transfer matrix based on the Baker-Campbell-Hausdorff (BCH) formula [39]

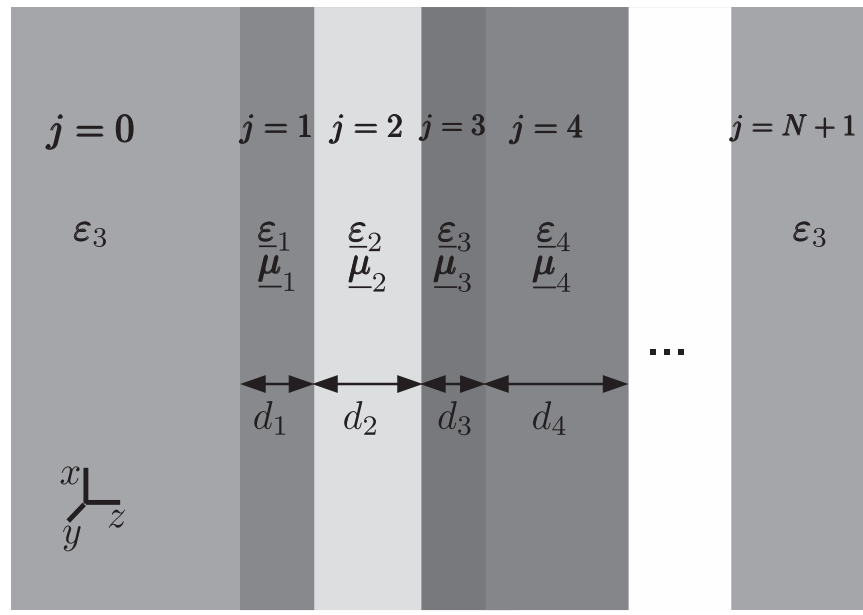

FIG. 1. One-dimensional stratification of $N$ finite thickness anisotropic dielectric slabs representing an arbitrary planar multilayer stack. The stratified media, $i=1, N$, with generally anisotropic layers are embedded in an isotropic bathing medium. Each layer has an arbitrary material composition, thickness, and orientation.

as a second-order expansion in this parameter $\delta$, which is inversely proportional to the pitch length of the cholesteric liquid crystal. The form of the transfer matrix is then obtained as a function of the ordinary and extraordinary eigenvalues of the Maxwell equations, averaged over the orientations in the multilayer system. Consequently, our solutions enable us to analyze the interaction between two semi-infinite homochiral or heterochiral layers and thus quantify macroscopically the effects of chirality. Our results point to a realistic possibility of measuring the effects of chirality in the macroscopic CasimirLifshitz torque for interaction geometries involving oriented cholesteric liquid crystals. Note that this is different from the effects of chirality of an intervening medium [40-42].

The paper is organized as follows. After the Introduction we will discuss the solutions of the Maxwell equations for a general planar anisotropic multilayer in Sec. II. Next we will introduce the simplification for the cholesteric liquid crystal configuration in Sec. III, the numerical implementation of which is then covered in Sec. IV. Conclusions can be found in Sec. V.

\section{PLANAR ANISOTROPIC MULTILAYER}

\section{A. Transfer matrix}

Here we will formulate the theory of Casimir-Lifshitz interactions in a system composed of a one-dimensional layering of finite thickness anisotropic dielectric slabs (see Fig. 1). Later on, we will use this geometry to model a cholesteric liquid crystal. Our approach is based on an application of the transfer matrix method for the electromagnetic field modes propagating through one-dimensional stratifications. This method was introduced as an algebra of 4 $\times 4$ matrices by Berreman [19] and later reformulated by Yeh [20]. Here we will follow the latter author's formulation, which is based on the change of basis transform in linear algebra. However, both formulations are equivalent [43]. We assume that the anisotropic stratification coincides 
with the direction of the cholesteric axis, oriented along the $z$-direction, and perpendicular to the translational $x-y$ plane of symmetry of the laboratory coordinate system (see Fig. 1).

We start by formulating Maxwell's equations as an eigenvalue problem, as we have done before for the case of single interfaces [24]. Assume that the electromagnetic (EM) wave propagates within one of the anisotropic slabs labeled as $j$ whose anisotropic plane is facing the surface, which is defined as the $x$ - $y$-plane in the laboratory coordinate system. Furthermore, the magnetic and electric anisotropy axes are assumed to be identical, implying that the electric permittivity and the magnetic permeability are given by

$$
\begin{array}{r}
\underline{\boldsymbol{\varepsilon}}_{j}(\omega)=\left(\begin{array}{ccc}
\varepsilon_{j x} \cos ^{2} \theta_{j}+\varepsilon_{j y} \sin ^{2} \theta_{j} & \left(\varepsilon_{j x}-\varepsilon_{j y}\right) \sin \theta_{j} \cos \theta_{j} & 0 \\
\left(\varepsilon_{j x}-\varepsilon_{j y}\right) \sin \theta_{j} \cos \theta_{j} & \varepsilon_{j x} \sin ^{2} \theta_{j}+\varepsilon_{j y} \cos ^{2} \theta_{j} & 0 \\
0 & 0 & \varepsilon_{j z}
\end{array}\right), \\
\underline{\boldsymbol{\mu}}_{j}(\omega)=\left(\begin{array}{ccc}
\mu_{j x} \cos ^{2} \theta_{j}+\mu_{j y} \sin ^{2} \theta_{j} & \left(\mu_{j x}-\mu_{j y}\right) \sin \theta_{j} \cos \theta_{j} & 0 \\
\left(\mu_{j x}-\mu_{j y}\right) \sin \theta_{j} \cos \theta_{j} & \mu_{j x} \sin ^{2} \theta_{j}+\mu_{j y} \cos ^{2} \theta_{j} & 0 \\
0 & 0 & \mu_{j z}
\end{array}\right),
\end{array}
$$

where $\theta_{j}$ denotes the angle between the optic axis of layer $j$ and the $x$-axis of the laboratory's coordinate system. It must be stressed that the entries of both $\underline{\boldsymbol{\varepsilon}}_{j}$ and $\underline{\boldsymbol{\mu}}_{j}$ depend on frequency, but that the argument will be suppressed from now on. Because of this dependence it is convenient to represent the electromagnetic fields in the frequency domain as well. After also transforming to Fourier space, the vectorial Maxwell equations are given by

$$
\mathbf{k} \times \mathbf{E}=\frac{\omega}{c} \mathbf{B}, \quad \mathbf{k} \times \mathbf{H}=-\frac{\omega}{c} \mathbf{D},
$$

where $\mathbf{B}=\boldsymbol{\mu} \cdot \mathbf{H}$ and $\mathbf{D}=\underline{\boldsymbol{\varepsilon}} \cdot \mathbf{E}$. Equation (3) is a system of six linear equations with six unknowns, four of which are independent. Elimination of the $z$ component of $\mathbf{E}$ and $\mathbf{H}$ leads to the following four-dimensional eigenvalue equation [44]:

$$
\underline{Q}_{j} \Psi=q \Psi,
$$

for the four-dimensional EM field vector $\Psi=\left(E_{x}, E_{y}, H_{x}, H_{y}\right)^{T} \cdot \underline{Q}_{j}$ denotes a $4 \times 4$ antidiagonal block matrix whose explicit entries are given in the Supplemental Material [45], Sec. I.

The four eigenvector solutions of Eq. (4) represent the orientation-dependent modes, which both can propagate forward and backward. They are denoted by $\Psi_{j o}^{ \pm}$and $\Psi_{j e}^{ \pm}$, where the subscripts $e$ and $o$ denote extraordinary and ordinary, respectively (for the case of birefringent media), and the superscripts + and - indicate forward and backward propagating modes, respectively. The mode eigenvalues are given by

$$
\begin{aligned}
& q_{j o}^{ \pm}= \pm \sqrt{\varepsilon_{j y} \mu_{j x} \omega^{2} / c^{2}-\left(\mu_{j x} / \mu_{j z}\right) k_{\rho}^{2} \cos ^{2}\left(\theta_{j}-\eta\right)-k_{\rho}^{2} \sin ^{2}\left(\theta_{j}-\eta\right)}, \\
& q_{j e}^{ \pm}= \pm \sqrt{\varepsilon_{j x} \mu_{j y} \omega^{2} / c^{2}-\left(\varepsilon_{j x} / \varepsilon_{j z}\right) k_{\rho}^{2} \cos ^{2}\left(\theta_{j}-\eta\right)-k_{\rho}^{2} \sin ^{2}\left(\theta_{j}-\eta\right)},
\end{aligned}
$$

where $k_{\rho}$ denotes the radial component of the wave vector and $\eta$ represents the azimuthal component of the EM wave propagation direction.

Here we formulate a $4 \times 4$ transfer matrix formalism for a one-dimensional stratified system composed planar slabs of finite thickness with in-plane anisotropy (see Fig. 1), based on the methodology found in Ref. [20].

The matrix in Eq. (4) has distinct eigenvalues so it is diagonalizable. This brings us to the so-called propagation matrix

$$
\begin{aligned}
\underline{\boldsymbol{P}}_{j} \equiv & \operatorname{diag}\left[\exp \left(-i q_{j e} d_{j}\right), \exp \left(i q_{j e} d_{j}\right), \exp \left(-i q_{j o} d_{j}\right),\right. \\
& \left.\exp \left(i q_{j o} d_{j}\right)\right] .
\end{aligned}
$$

Multiplication from the left of the matrix $\underline{\boldsymbol{P}}_{j}$ by the EM field vector represents propagation within an anisotropic slab in the $z$-direction over a distance $d_{j}$, the thickness of the $j$ th layer

$$
\boldsymbol{\Psi}\left(z+d_{j}\right)=\underline{\boldsymbol{P}}_{j} \cdot \boldsymbol{\Psi}(z),
$$

where $\boldsymbol{\Psi}$ again denotes a four-dimensional EM wave vector. $\underline{\boldsymbol{P}}_{j}$ is a valid representation only in the eigenvector basis where the matrix is diagonal. Hence it is transformed to the $x y$ basis as follows:

$$
\exp \left(-i \underline{\boldsymbol{Q}}_{j} d_{j}\right)=\underline{\boldsymbol{S}}_{j} \cdot \underline{\boldsymbol{P}}_{j} \cdot \underline{\boldsymbol{S}}_{j}^{-1},
$$

where the columns of $\underline{S}_{j}$ consist of the eigenvectors of Maxwell's equations

$$
\underline{\boldsymbol{S}}_{j}=\left(\begin{array}{cccc}
\mid & \mid & \mid & \mid \\
\boldsymbol{\Psi}_{j e}^{+} & \boldsymbol{\Psi}_{j e}^{-} & \boldsymbol{\Psi}_{j o}^{+} & \boldsymbol{\Psi}_{j o}^{-} \\
\mid & \mid & \mid & \mid
\end{array}\right)
$$


which is analogous to what is called the "rotation matrix" in Ref. [35]. In Eq. (8) the normalization constants of the eigenvectors can be omitted because $\underline{\boldsymbol{P}}_{j}$ is multiplied by the inverse of $\underline{\boldsymbol{S}}_{j}$ from the right. The total transfer matrix that represents the EM wave propagation through all $N$ layers is given by

$$
\begin{aligned}
\underline{\boldsymbol{T}}_{N} \equiv & \ldots \underline{\boldsymbol{S}}_{j-1} \cdot \underline{\boldsymbol{P}}_{j-1} \cdot \underline{\boldsymbol{S}}_{j-1}^{-1} \cdot \underline{\boldsymbol{S}}_{j} \cdot \underline{\boldsymbol{P}}_{j} \cdot \underline{\boldsymbol{S}}_{j}^{-1} \cdot \underline{\boldsymbol{S}}_{j+1} \\
& \underline{\boldsymbol{P}}_{j+1} \cdot \underline{\boldsymbol{S}}_{j+1}^{-1} \ldots \equiv \prod_{j=1}^{N} \underline{\boldsymbol{S}}_{j} \cdot \underline{\boldsymbol{P}}_{j} \cdot \underline{\boldsymbol{S}}_{j}^{-1},
\end{aligned}
$$

where the product symbol denotes matrix multiplication from the right for each subsequent index.

Next we have to consider what happens outside the slab, i.e., the cases $j=0$ and $j=N+1$. The EM field propagating through the isotropic embedding medium can be written as a linear combination of the $s$ - and $p$-polarized modes. By the same token as before, since the $s$ - and $p$-polarized modes are the eigenmodes for isotropic media, a matrix can be constructed with the $s$ - and $p$-polarized mode eigenvectors as columns for $j=0$ :

$$
\underline{\boldsymbol{S}}_{0}=\left(\begin{array}{cccc}
\mid & \mid & \mid & \mid \\
\boldsymbol{\Psi}_{j s}^{+} & \boldsymbol{\Psi}_{j s}^{-} & \boldsymbol{\Psi}_{j p}^{+} & \boldsymbol{\Psi}_{j p}^{-} \\
\mid & \mid & \mid & \mid
\end{array}\right)
$$

the explicit form of which can be found in the Supplemental Material [45], Sec. I. However, the case $j=N+1$ is a little different. Here only forward-propagating modes exist, which does not affect the matrix itself but instead only affects the vector on which the matrix acts. After all, in the $s$ - and $p$-mode basis, this information can be represented by two nonzero components, which represent the amplitudes of the forward propagating $s$ - and $p$-polarized modes, (see Sec. II B for details). Note that the matrices of Eqs. (8) and (10) are singular at zero frequency. However, physically "zero frequency" corresponds to a static limit where the frequency is small on the scale of the main absorption frequency of the materials. Hence within this limit it is possible to obtain the inverses of the matrices of Eqs. (8) and (10). See also Supplemental Material [45], Sec. V for more details on how to take the nonretarded limit.

Since Eq. (9) is valid in the laboratory $x y$ basis, it must be transformed to the $s p$-mode basis, which is the proper eigenmode basis for isotropic media. The total transfer matrix is hence

$$
\underline{\boldsymbol{M}}=\underline{\boldsymbol{S}}_{0}^{-1} \cdot \underline{\boldsymbol{T}}_{N} \cdot \underline{\boldsymbol{S}}_{0},
$$

where $\underline{\boldsymbol{T}}_{N}$ is given by Eq. (9). For the general case considered here it is not possible to give an explicit closed expression for the matrix $\underline{\boldsymbol{M}}$. As a test case, let us consider the limit of an infinitely thick slab. This limit does not directly and algebraically follow from Eq. (11) (see Supplemental Material [45], Sec. III for details). It can be shown that for a semiinfinite slab, the transfer matrix simplifies to

$$
\underline{M}=\underline{S}_{0}^{-1} \cdot \underline{S}_{1} .
$$

\section{B. Lifshitz formula}

The Casimir-Lifshitz interaction free energy can be obtained from the secular determinant of the modes whose zeros give the eigenfrequencies of the bound states [35]. However, in its turn the secular determinant itself can be rewritten in terms of the Fresnel reflection coefficients that can be obtained from the transfer matrix formalism. This route then connects the transfer matrix formalism with the Casimir-Lifshitz interactions, which we elucidate in what follows. Grosso modo one can follow either the approach based on the Green's function tensors, closest to the original papers by Lifshitz et al. [2] (see, e.g., Refs. [46-48]) or follow the heuristic derivation by van Kampen et al. [49,50], which was later shown to be actually exact [51], and based on the summation over the allowed EM modes within a given geometry, more akin to the original paper by Casimir [1]. It can be shown that both approaches lead to the same result [52], namely the Lifshitz formula for dispersion interactions. Here we will take advantage of this result.

We assume that the matrix $\underline{\boldsymbol{M}}$ from Eq. (11) is known and that its entries are given by $\underline{\boldsymbol{M}}=\left(M_{i k}\right)$ with $i, k \in\{1,2,3,4\}$. In such a case, the Fresnel reflection matrix elements can be shown to be given by $[20,43]$

$$
\begin{aligned}
& r_{s s}=\frac{M_{21} M_{33}-M_{23} M_{31}}{M_{11} M_{33}-M_{13} M_{31}}, \\
& r_{s p}=\frac{M_{33} M_{41}-M_{31} M_{43}}{M_{11} M_{33}-M_{13} M_{31}}, \\
& r_{p s}=\frac{M_{11} M_{23}-M_{13} M_{21}}{M_{11} M_{33}-M_{13} M_{31}}, \\
& r_{p p}=\frac{M_{11} M_{43}-M_{13} M_{41}}{M_{11} M_{33}-M_{13} M_{31}} .
\end{aligned}
$$

We verified that combining Eq. (12) with Eq. (13) restores the known Fresnel reflection matrix [21,24] for a semi-infinite birefringent plate.

To determine the Casimir energy and torque, (13a) to (13d) must be inserted into the Lifshitz formula. The energy per unit area is given by

$$
\begin{aligned}
\frac{E_{\mathrm{Cas}}}{A}= & \frac{k_{b} T}{4 \pi^{2}} \sum_{n=0}^{\infty}\left(1-\frac{1}{2} \delta_{0 n}\right) \int_{0}^{\infty} \int_{0}^{2 \pi} \ln \left[D\left(k_{\rho}, \eta,\left\{\theta_{j}\right\}, \varphi, i \zeta_{n}\right)\right] \\
& \times k_{\rho} d k_{\rho} d \eta,
\end{aligned}
$$

where [47]

$$
D=\operatorname{det}\left[\underline{\boldsymbol{I}}-\underline{\boldsymbol{r}}_{1}\left(\left\{\theta_{j}\right\}, i \zeta_{n}\right) \cdot \underline{\boldsymbol{r}}_{2}\left(\theta_{1}+\varphi, i \zeta_{n}\right) e^{-2 k_{3} a}\right],
$$

and the reflection matrices are given by

$$
\underline{\boldsymbol{r}}_{q}=\left(\begin{array}{ll}
r_{q, s s} & r_{q, s p} \\
r_{q, p s} & r_{q, p p}
\end{array}\right), \quad q \in\{1,2\} .
$$

The subscript $q$ is merely a label for each multilayer stack. The entries of the matrices are given by (13a) to (13d). All quantities are evaluated at the imaginary Matsubara frequencies $\zeta_{n}=\frac{2 \pi n k_{b} T}{\hbar}$ so that each contribution to the Casimir energy decreases monotonically with $n$. The Casimir torque is then given by

$$
\tau(a, \varphi)=-\frac{\partial E_{\mathrm{Cas}}}{\partial \varphi},
$$




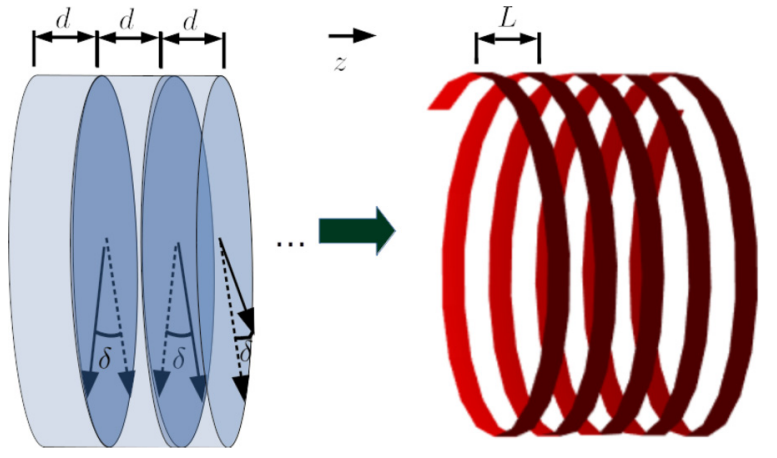

FIG. 2. Illustration of the continuum approximation used here to model a liquid crystal. It consists of layers with identical thicknesses $d$ but each with a slightly different orientation, each with identical increments $\delta \ll 1$. Consequently, the multilayer structure manifests as a continuous inhomogeneity in the direction perpendicular to the plane of reflection (defined as the $z$-direction), where the orientation effectively behaves as a helix with pitch length $L$.

where $\varphi$ denotes the angle between the optic axes of the layers of each stack closest to the gap. This result is equivalent to that of Ref. [35], which uses the formalism of Berreman [19], while here the same formalism has been used with the basis suggested by Yeh [20].

\section{MODELING A CHOLESTERIC LIQUID CRYSTAL}

A cholesteric liquid crystal is modeled as a nonmagnetic uniaxial planar multilayer stack. All anisotropic layers of equal thickness $d$ are made of identical materials, and only differ by their orientation $\theta_{j}$. We start by writing Eq. (7) as

$$
\exp \left(-i \underline{\boldsymbol{Q}}_{j} d\right)=\underline{\boldsymbol{S}}_{j} \cdot \underline{\boldsymbol{P}}_{j} \cdot \underline{\boldsymbol{S}}_{j}^{-1}
$$

for a single slab with label $j$.

Now let us formulate the conditions of validity of this approximation. In accordance with an actual cholesteric liquid crystal [34], each layer is assumed to be infinitesimally thin, i.e., $d \rightarrow 0$. Moreover, let the difference in orientation between each subsequent layer $\delta$ also be infinitesimally small, in such a way that this quantity parametrically describes a helix in space (see Fig. 2). Consequently, an infinitesimal change in $z$ within the liquid crystal is proportional to an infinitesimal change in the orientation:

$$
d z=\mathrm{d}=\frac{L}{2 \pi} \delta,
$$

where $L$ denotes the pitch length, in which the orientations of the layers complete one period, and $\delta \ll 1$. Hence there is some freedom of choice, we can use either $z$ or $\theta$ as a (continuous) variable. In what follows we will use two variables interchangeably. Another assumption is that the total thickness $N d$, where $N$ denotes the total number of layers, is infinite. This is justifiable because a typical thickness of liquid crystals is of the order of several microns, and we will limit ourselves to separation distances of less than one micron $(a \ll N d)$, where the Casimir-Lifshitz torque is likely to be large enough to be measured [29]. Finally, it will be assumed that the pitch length of the cholesteric liquid crystal $L$, in which the orientations of the layers complete one period, is smaller than the total thickness. Hence at least one period is assumed to fit inside the crystal. We can summarize the condition for our approximation as follows:

$$
d \ll L \ll N d
$$

which means that the difference between the first orientation and last orientation is at least $2 \pi$ radians. This is applicable to a cholesteric liquid crystal [34]. We also require $d$ to be small on the scale of the matrix norm of $\underline{Q}_{j}$ :

$$
\left\|\underline{\boldsymbol{Q}}_{j}\right\| d \ll 1 \text { for all } 1 \leqslant j \leqslant N .
$$

Physically, Eq. (20) can be understood as the thickness $d$ being much smaller than the typical values of the relevant wavelengths. After all, in a continuous medium approach, the relevant wavelengths are assumed to be larger than the typical distances between atoms. Since the physical thickness is actually of the same order of magnitude, it can be approximated as a numerical integration stepsize in the context of a continuous medium approach. The condition Eq. (20) allows us to use the Baker-Campbell-Hausdorff (BCH) formula [39]. The second condition of Eq. (19) allows us to use Eq. (12) instead of Eq. (11).

In Sec. IV of the Supplemental Material [45] we derive the following expression valid up to the second order in the angular deviation in the orientation between the neighboring uniaxial layers:

$$
\begin{aligned}
\prod_{j=1}^{N} \exp \left(-i d \underline{\boldsymbol{Q}}_{j}\right) & =\exp \left(-i d \sum_{j=1}^{N} \underline{\boldsymbol{Q}}_{j}+O\left(\delta^{2}\right)\right) \\
& \longrightarrow \exp \left(-i \int_{\min (z)}^{\max (z)} \underline{\boldsymbol{Q}}(\theta) d z+O\left(\delta^{2}\right)\right),
\end{aligned}
$$

where eventually we assumed that $j$ changes as a continuous variable associated with $z$. The full derivation is somewhat lengthy so we will summarize it here. In short, we use the Baker-Campbell-Haussdorff (BCH) formula [39] up to third order in $\delta$. Then we combine the $\mathrm{BCH}$ formula with finite difference coefficients [53] to determine that three nearest neighbors to the right are needed to obtain the same accuracy as the $\mathrm{BCH}$ formula.

To evaluate the argument of the exponential matrix in Eq. (21) we will try to determine its diagonalized form. Let us focus on the leading-order term $-i d \sum_{j=1}^{N} \underline{Q}_{j}$. Even though Eq. (7) tells us that each individual matrix $\underline{\boldsymbol{Q}}_{j}$ is diagonalizable, it does not follow in general that their sum is also diagonalizable. However, within this particular approximation it is assumed that the commutators between the matrices $\underline{\boldsymbol{Q}}_{j}$ are negligible and the matrices effectively commute, with the error of this approximation being of second order. Commuting diagonalizable matrices can be shown to have the same eigenvectors. Consequently, each diagonalizable matrix $\underline{Q}_{j}$ can be transformed to the same eigenvector basis via one matrix, say $\underline{\boldsymbol{S}}$. In other words, the matrices $\underline{\boldsymbol{Q}}_{j}$ can be simultaneously 
diagonalized

$$
\sum_{j=1}^{N} \underline{\boldsymbol{Q}}_{j}=\underline{\boldsymbol{S}} \cdot \underline{\boldsymbol{D}} \cdot \underline{\boldsymbol{S}}^{-1},
$$

where $\underline{\boldsymbol{D}}$ is a diagonal matrix. This furthermore implies that

$$
\exp \left(-i d \sum_{j=1}^{N} \underline{\boldsymbol{Q}}_{j}\right)=\underline{\boldsymbol{S}} \exp \left[-i d \underline{\boldsymbol{D}}+O\left(d^{2}\right)\right] \underline{\boldsymbol{S}}^{-1} .
$$

We know that the matrices $\boldsymbol{S}$ and $\boldsymbol{D}$ exist but now we want to determine their explicit form. The physical interpretation of Eq. (22) is that, in this approximation, the orientations of the different layers are averaged over the entire crystal. Since $d \rightarrow 0$ and $N \rightarrow \infty$, it can be proposed that $d \sim 1 / N$, and the left-hand side of Eq. (22) becomes an average over all eigenvalues. Hence it follows that

$$
\underline{\boldsymbol{D}}=\frac{1}{N} \sum_{j=1}^{N} \operatorname{diag}\left\{q_{e}\left[\left(\theta_{j}-\eta\right)\right],-q_{e}\left[\left(\theta_{j}-\eta\right)\right], q_{o},-q_{o}\right\},
$$

which changes to an average with respect to a continuous distribution where the sum is replaced by an integral. The approximation of Eq. (23) is of course simple and crude, its error being of second order in the stepsize $d$. It assumes that the planar multilayered system can be approximated as a single slab with an averaged orientation. Essentially the effect of the presence of multiple layers is treated additively in this case.

The transfer matrix for such a configuration is given by [see Eq. (22)]

$$
\exp \left(-i d \sum_{j=1}^{N} \underline{\boldsymbol{Q}}_{j}\right)=\langle\underline{\boldsymbol{S}}\rangle \underline{\boldsymbol{P}}_{\mathrm{tot}}\left(\left\langle q_{e}\right\rangle\right)\langle\underline{\boldsymbol{S}}\rangle^{-1},
$$

where

$$
\begin{aligned}
\underline{\boldsymbol{P}}_{\mathrm{tot}}\left(\left\langle q_{e}\right\rangle\right)= & \operatorname{diag}\left[\exp \left(-i d_{\mathrm{tot}}\left\langle q_{e}\right\rangle\right), \quad \exp \left(i d_{\mathrm{tot}}\left\langle q_{e}\right\rangle,\right.\right. \\
& \left.\exp \left(-i d_{\mathrm{tot}} q_{o}\right), \quad \exp \left(i d_{\mathrm{tot}} q_{o}\right)\right] .
\end{aligned}
$$

Here $d_{\text {tot }}=N d$ represents the total thickness, and in the limit $d_{\text {tot }} \rightarrow \infty, \underline{\boldsymbol{P}}_{\text {tot }}$ tends to $\underline{\boldsymbol{P}}_{\text {tot }} \rightarrow \operatorname{diag}(1,0,1,0)$. (This does not follow directly algebraically, but from physical considerations, see Supplemental Material [45], Sec. III). In Eq. (25) the eigenvectors are the ones corresponding to $\left\langle q_{e}\right\rangle$ :

$$
\langle\underline{\boldsymbol{S}}\rangle=\underline{\boldsymbol{S}}\left(\left\langle q_{e}\right\rangle, \quad\langle\theta\rangle\right),
$$

where $\underline{S}$ is given by Eq. (8) and $\left\langle q_{e}\right\rangle$ denotes the extraordinary eigenvalue, averaged over the orientations

$$
\left\langle q_{e}\right\rangle=\frac{1}{N} \sum_{j=1}^{N} q_{e}\left[\left(\theta_{j}-\eta\right)\right] \longrightarrow \frac{1}{N \delta} \int_{0}^{N \delta} q_{e}(\theta-\eta) d \theta .
$$

The rightmost expression follows after the continuity approximation that allows us to replace the sums over the discrete index $j$ by integrals over the continuous variable $\theta$.

Due to the periodicity of $q_{e}, N \delta$ can be replaced by $\pi$, so that $q_{e}$ is averaged over one period and it can take all possible real values. This is because the thickness of the liquid crystal is assumed to be larger than the pitch length [see Eq. (19)]. Consequently, at least one period can be fit into the crystal. The integer part of the ratio between the thickness and the pitch length will dominate the remainder because the remainder represents a part of the liquid crystal located at an infinite distance from the plane of reflection. Setting $N \delta=\pi$ also allows us to set $\eta=0$ because the integral becomes translation invariant over one period. In this case, the integral of Eq. (27) can be evaluated analytically

$$
\begin{aligned}
\left\langle q_{e}\right\rangle & =\frac{1}{\pi} \int_{0}^{\pi} q_{e}(\theta) d \theta \\
& = \pm \frac{2}{\pi} \sqrt{\varepsilon_{1 x}\left(\frac{k_{\rho}^{2}}{\varepsilon_{1 y}}+\frac{\zeta^{2}}{c^{2}}\right)} E\left(\frac{k_{\rho}^{2}\left(\frac{\varepsilon_{1 x}}{\varepsilon_{1 y}}-1\right)}{\varepsilon_{1 x}\left(\frac{k_{\rho}^{2}}{\varepsilon_{1 y}}+\frac{\zeta^{2}}{c^{2}}\right)}\right),
\end{aligned}
$$

where $E(z)$ denotes the complete elliptic integral of the second kind, given by [54]

$$
E(z)=\int_{0}^{\frac{\pi}{2}} \sqrt{1-z \sin ^{2} t} d t
$$

the value of which is real for arguments $-1 \leqslant z \leqslant 1$.

To determine the average orientation, we must solve the following equation:

$$
\left\langle q_{e}\right\rangle= \pm \sqrt{\varepsilon_{1 x} \frac{\zeta^{2}}{c^{2}}+\frac{\varepsilon_{1 x}}{\varepsilon_{1 y}} k_{\rho}^{2} \cos ^{2}\langle\theta\rangle+k_{\rho}^{2} \sin ^{2}\langle\theta\rangle},
$$

which has four possible solutions

$$
\langle\theta\rangle= \pm \arccos \left( \pm \frac{\sqrt{k_{\rho}^{2}+\varepsilon_{1 x} \zeta^{2} / c^{2}-\left\langle q_{e}\right\rangle^{2}}}{k_{\rho} \sqrt{1-\frac{\varepsilon_{1 x}}{\varepsilon_{1 y}}}}\right) .
$$

Here the different signs of $\langle\theta\rangle$ represent the different chiralities of the liquid crystal: a positive $\langle\theta\rangle$ is associated with right handed chirality and the minus sign corresponds to left-handed chirality, if the axial wave vector component is positive. The different signs in the argument of the arc-cosine in Eq. (30) come from the different square roots in Eq. (29), associated with the two different propagation directions of the extraordinary modes in the liquid crystal. However, since the quantity $\left\langle q_{e}\right\rangle$ is an average along the helix, its sign must be interpreted as the direction of the helical spiral. Alternatively, the different signs of the square root of $\left\langle q_{e}\right\rangle$ in Eq. (29) can be thought of as the location of the crystal: since the thicknesses of the crystals are assumed to be infinite, the negative square root represents the crystal being on the left and the positive square root represents the crystal being on the right. This distinction depends on the arbitrary choice of coordinates and it is hence not physical. Equation (30) provides four solutions, whereas there are only two physically distinguishable shapes of the helix, namely left- and right-handed. Therefore, we need to establish how they can be physically interpreted. We require that the physical $\langle\theta\rangle$ is real. First, we have to verify that such solutions exist. To this end we distinguish two cases: $\varepsilon_{1 y} \geqslant \varepsilon_{1 x}$ and $\varepsilon_{1 y} \leqslant \varepsilon_{1 x}$. In order for $\langle\theta\rangle$ to be real, we need to check that

$$
0 \leqslant k_{\rho}^{2}+\varepsilon_{1 x} \frac{\zeta^{2}}{c^{2}}-\left\langle q_{e}\right\rangle^{2} \leqslant k_{\rho}^{2}\left(1-\frac{\varepsilon_{1 x}}{\varepsilon_{1 y}}\right),
$$

which can be seen to be the case since

$$
\min \left(1, \frac{\varepsilon_{1 x}}{\varepsilon_{1 y}}\right) \leqslant \frac{\varepsilon_{1 x}}{\varepsilon_{1 y}} \cos ^{2} \theta+\sin ^{2} \theta \leqslant \max \left(1, \frac{\varepsilon_{1 x}}{\varepsilon_{1 y}}\right)
$$


for all real $\theta$. Here it is assumed that $|\langle\theta\rangle| \leqslant \pi$. Hence the real value of the average within the domain $-\pi \leqslant\langle\theta\rangle \leqslant \pi$ orientation is

$$
\begin{aligned}
\langle\theta\rangle_{L} & =\arccos \left(-\frac{\sqrt{k_{\rho}^{2}+\varepsilon_{1 x} \zeta^{2} / c^{2}-\left\langle q_{e}\right\rangle^{2}}}{k_{\rho} \sqrt{1-\frac{\varepsilon_{1 x}}{\varepsilon_{1 y}}}}\right), \\
\langle\theta\rangle_{R} & =-\arccos \left(-\frac{\sqrt{k_{\rho}^{2}+\varepsilon_{1 x} \zeta^{2} / c^{2}-\left\langle q_{e}\right\rangle^{2}}}{k_{\rho} \sqrt{1-\frac{\varepsilon_{1 x}}{\varepsilon_{1 y}}}}\right),
\end{aligned}
$$

if the crystal is on the left and

$$
\langle\theta\rangle_{L}=-\arccos \left(\frac{\sqrt{k_{\rho}^{2}+\varepsilon_{1 x} \zeta^{2} / c^{2}-\left\langle q_{e}\right\rangle^{2}}}{k_{\rho} \sqrt{1-\frac{\varepsilon_{1 x}}{\varepsilon_{1 y}}}}\right),
$$

$$
\langle\theta\rangle_{R}=\arccos \left(\frac{\sqrt{k_{\rho}^{2}+\varepsilon_{1 x} \zeta^{2} / c^{2}-\left\langle q_{e}\right\rangle^{2}}}{k_{\rho} \sqrt{1-\frac{\varepsilon_{1 x}}{\varepsilon_{1 y}}}}\right),
$$

if the crystal is on the right. Here $\left\langle q_{e}\right\rangle$ is given by Eq. (28). The subscripts $L$ and $R$ in Eqs. (32) and (31) denote the left- and right-handedness of the liquid crystal, respectively. Note that Eqs. (32) and (31) depend on frequency, which in turn affects the frequency dependence of the reflection matrix. Finally, Eqs. (28), (32), and (31) have to be inserted into Eq. (8) to obtain $\langle\underline{\boldsymbol{S}}\rangle_{1}$.

Now we move on to the next-order term in the $\mathrm{BCH}$ expansion. Here the contribution of nested commutators is neglected, i.e., commutators are assumed to commute with other matrices. To take advantage of Eq. (24) we apply the $\mathrm{BCH}$ formula again to separate the $\mathrm{BCH}$ terms as follows (see Supplemental Material [45], Sec. IV for a detailed derivation):

$$
\begin{gathered}
\exp \left(-i \frac{L}{\pi} \int_{0}^{N \delta} \underline{\boldsymbol{Q}}(\theta) d \theta-3 \frac{L^{2}}{\pi^{2}} \int_{0}^{(N-3) \delta}\left[\underline{\boldsymbol{Q}}(\theta), \frac{\partial \underline{\boldsymbol{Q}}(\theta)}{\partial \theta}\right] d \theta+O\left(\delta^{3}\right)\right) \\
=\langle\underline{\boldsymbol{S}}\rangle_{1} \underline{\boldsymbol{P}}_{\mathrm{tot}}\left(\left\langle q_{e}\right\rangle\right)\langle\underline{\boldsymbol{S}}\rangle_{1}^{-1} \exp \left(-3 \frac{L^{2}}{\pi^{2}} \int_{j=1}^{(N-3) \theta}\left[\underline{\boldsymbol{Q}}(\theta), \frac{\partial \underline{\boldsymbol{Q}}(\theta)}{\partial \theta}\right] d \theta\right),
\end{gathered}
$$

where we took advantage of the transfer matrix for the single interface case, Eq. (12). The argument of rightmost exponential matrix on the right-hand side of Eq. (33) is not jointly diagonalizable with the first $\mathrm{BCH}$ term [55]. However, this correction is expected to be small compared to the averaged factor on its left. This allows us to write it as a Taylor expansion. Hence we obtain

$$
\prod_{j=1}^{N} \exp \left(-i d \underline{\boldsymbol{Q}}_{j}\right)=\langle\underline{\boldsymbol{S}}\rangle_{1} \underline{\boldsymbol{P}}_{\mathrm{tot}}\left(\left\langle q_{e}\right\rangle\right)\langle\underline{\boldsymbol{S}}\rangle_{1}^{-1}\left(\underline{\boldsymbol{I}}-3 \frac{L^{2}}{\pi^{2}} \int_{0}^{(N-3) \delta}\left[\underline{\boldsymbol{Q}}(\theta), \frac{\partial \underline{\boldsymbol{Q}}(\theta)}{\partial \theta}\right] d \theta+O\left(\delta^{3}\right)\right) .
$$

The leading-order term in Eq. (34) can be understood as a slab with infinite thickness and an averaged orientation. The secondorder correction describes the effect due to the finite thicknesses of the layers and their slightly different orientations. For a liquid crystal of infinite thickness Eq. (34) becomes

$$
\prod_{j=1}^{N} \exp \left(-i d \underline{\boldsymbol{Q}}_{j}\right)=\underline{\boldsymbol{S}}_{0}^{-1}\left\langle\underline{\boldsymbol{S}}_{1}\left(\underline{\boldsymbol{I}}-3 \frac{L^{2}}{\pi^{2}} \int_{0}^{(N-3) \delta}\left[\underline{\boldsymbol{Q}}(\theta), \frac{\partial \underline{\boldsymbol{Q}}(\theta)}{\partial \theta}\right] d \theta+O\left(\delta^{3}\right)\right) .\right.
$$

Next we are faced with the task of writing Eq. (35) as an expansion in $\delta$. Note that the integral absorbs one factor of $\delta$ and that the integration from 0 to $N \delta=\pi$ will not contribute to the integral (see Supplemental Material [45], Sec. I for the explicit expressions for the commutator), so the integral is expected to be small $\sim \delta$. Within the approximations used here, it suffices to calculate the integral up to first order in $\delta$. (A more precise calculation would be beyond the approximation and hence be inconsistent). We will denote this first-order coefficient by $\boldsymbol{A}$. Hence the transfer matrix is

$$
\prod_{j=1}^{N} \exp \left(-i d \underline{\boldsymbol{Q}}_{j}\right)=\underline{\boldsymbol{S}}_{0}^{-1}\langle\underline{\boldsymbol{S}}\rangle_{1}\left(\underline{\boldsymbol{I}}-3 \frac{L^{2}}{\pi^{2}} \underline{\boldsymbol{A}} \delta+O\left(\delta^{3}\right)\right)
$$

where $\underline{\boldsymbol{A}}$ is given by the integral in Eq. (35), and its explicit expressions can be found in the Supplemental Material [45], Sec. I. Note that the error of the $\mathrm{BCH}$ formula is of third order in $\delta$, as the higher-order term Eq. (36) is actually quadratic in $\delta$. So this term does not have to follow the sign convention of Eqs. (31) and (32). Since the approximate transfer matrix is known now, Eq. (13) can be applied to it to obtain the corresponding Fresnel matrix. Note that the factor of $L^{2}$ cancels out of the higher-order correction for the Fresnel matrix. However, it is worth pointing out that $\delta$ is inversely proportional to $L$ [see Eq. (18)], so that the final result still depends on the pitch length. We again refer to the Supplemental Material [45], Sec. I for the explicit expressions for the corrections to the Fresnel matrix. It is worth noting that the Fresnel matrices are invariant under $\boldsymbol{k} \rightarrow-\boldsymbol{k}$, hence they are identical whether they are placed on the left or on the right (if they have the same orientation). However, in general this is not necessarily true [56].

\section{RESULTS}

Here we will use the results derived in Sec. III to calculate the Casimir-Lifshitz torque given by Eq. (17). The first config- 
uration we consider is composed of a birefringent half-space, a finite vacuum gap of thickness $10 \mathrm{~nm}<a<1 \mu \mathrm{m}$, and a cholesteric half-space. Next we will investigate the combination of two cholesteric liquid crystals. The first in general should be accessible to experiments [29]. While realistically, surface roughness generally plays a significant role at this range [58], the roughness effects were considered elsewhere in detail [59-61] and we will ignore them here. We verified our calculations by reproducing earlier results obtained by implementations of the Barash formula $[32,62,63]$ (see Supplemental Material [45], Sec. V).

To calculate Casimir-Lifshitz interactions, the frequencydependent dielectric function of the interacting materials must be known. This is why we introduce it here first. We will ignore the temperature dependence of the dielectric function and assume a constant temperature of $293 \mathrm{~K}$. This is because we are interested in the cholesteric phase only.

The dielectric function of a nematic liquid crystal can be described by a semi-empirical three band model [64]

$$
\varepsilon_{N, x, y}(i \zeta)=\left(1+\sum_{k=1}^{3} \frac{C_{k, x, y}}{1+\frac{\zeta^{2}}{\omega_{k}^{2}}}\right)^{2} .
$$

However, a pure 5CB crystal is nematic and not cholesteric. It needs to be doped to reach a cholesteric phase, which is typically described by a Debye model [65]

$$
\varepsilon_{D, x, y}(i \zeta)=\varepsilon_{\infty, x, y}+\frac{\varepsilon_{x, y}(0)-\varepsilon_{\infty, x, y}}{1+\zeta \tau} .
$$

Therefore we propose to model a doped nematic liquid crystal as the sum of these dielectric functions

$$
\varepsilon_{x, y}(i \zeta)=\varepsilon_{D, x, y}(i \zeta)+\varepsilon_{N, x, y}(i \zeta) .
$$

However, the Debye relaxation processes take place on a much smaller frequency scale than the Matsubara frequencies at room temperature. More precisely, the smallest Matsubara frequency $(n=1)$ at $293 \mathrm{~K}$ is six orders of magnitude larger than the typical values of the Debye relaxation frequencies $[57,65]$. Therefore we propose to approximate it as the static term from Eq. (39)

$$
\varepsilon_{x, y}(i \zeta) \approx \varepsilon_{D, x, y}(0)+\varepsilon_{5 \mathrm{CB}, x, y}(i \zeta),
$$

in other words, the Debye processes only affect the static permittivity of the liquid crystal. The different components of the dielectric functions for both the nematic and cholesteric case in Fig. 3.

Now we are in a position to specify the materials, i.e., assign values to the parameters in Eq. (40). Let the cholesteric liquid crystal consist of 96 mass \% nematic 5CB (4- cyano4'-pentyl-biphenyl) doped with 4 mass \% chiral dopant S811 [57]. The value of the static Debye term for this mixture was taken from a recent experiment [57]. The dielectric function of 5CB was established in Ref. [33] based on data from Ref. [66]. The optical data of barium titanate $\left(\mathrm{BTO} \mathrm{BaTiO}_{3}\right)$ [67] will be used to describe the semi-infinite birefringent half-space. For more details about the parameter values and their effect on the Casimir-Lifshitz interaction we refer to Sec. VI of the Supplemental Material [45]. Throughout this section we choose the value of $\delta$ (if nonzero) to be 0.03 , which corresponds to a pitch

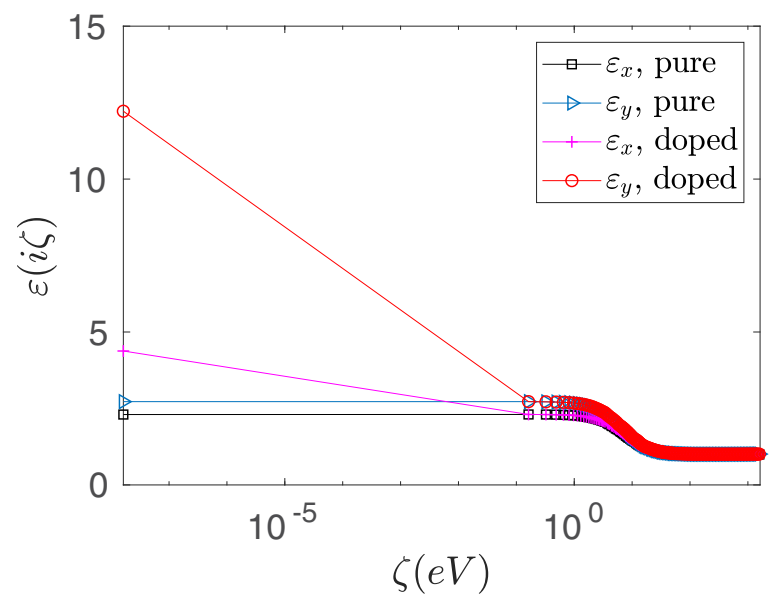

FIG. 3. Dielectric functions of pure, nematic, and doped, cholesteric 5CB. The dielectric function of nematic $5 \mathrm{CB}$ as proposed in Ref. [33] (indicated by the black squares and blue triangles) is modified to account for the presence of a chiral dopant. This affects the static values (see the magenta crosses and the red circles), which were obtained from Ref. [57].

length of $200 \mathrm{~nm}$ [see Eq. (18)]. This case will be indicated by the word "perturbative."

The results are shown in Fig. 4. The upper two panels in Figs. 4(a) and 4(b) display the two cases we distinguish here: the $\mathrm{BaTiO}_{3}$ crystal can be faced with either a right-handed or a left-handed crystal. Figure 4(c) shows the Casimir torque as a function of the misalignment angle $\varphi$. For reference, we included the usual case for a nematic liquid crystal, indicated by the cyan curve with triangles pointing to the right. The leading-order term in Eq. (36) creates a shift in the angular dependence of the torque of about $\pi / 2$ radians, making the proportionality closer to $\cos 2 \varphi$ than the usual $\sin 2 \varphi$. The sign of the torque changes depending on the left- or right-handedness of the cholesteric liquid crystal, which is a qualitative chirality effect. In other words, the torque follows the direction of the helix. While the leading-order term horizontally shifts the graph, the next-order term enhances the amplitude in only one direction, creating a clear deviation from the usual sinusoidal shape of the curve (even though it is still periodic with period $\pi$ ). For this reason we verified that

$$
\int_{0}^{\pi} \tau d \varphi=0
$$

so that no energy is gained or lost during a rotation over a period. Note that the shape of the curve is unlikely to be attributed to retardation effects, which are less significant at a distance of $10 \mathrm{~nm}$ than at larger separations. The higher-order correction actually is most significant at short distances $\sim 10 \mathrm{~nm}$, whereas retardation effects become more pronounced at larger distances. Even though the full retarded calculation was performed here, it turns out that retardation and finite pitch length effects do not correlate with each other. Finally, Fig. 4(d) shows the distance dependence of the amplitude of the torque. Not only is the amplitude enhanced significantly compared to the nematic case, it also decreases more slowly as a function of distance. Since the configuration of a birefringent and a semi-infinite liquid crystalline 


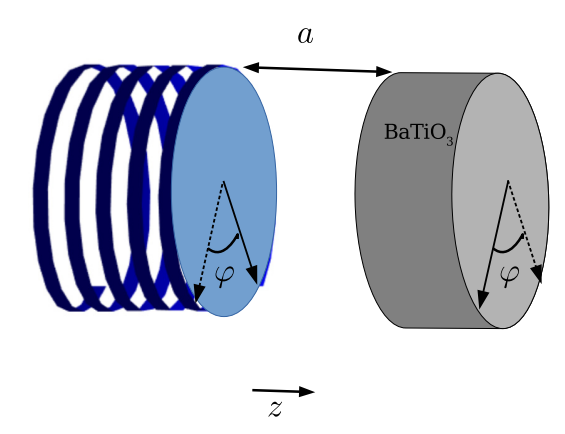

(a)

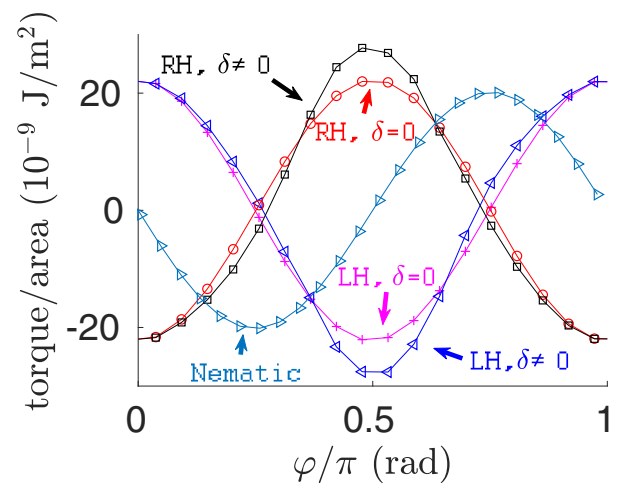

(c)

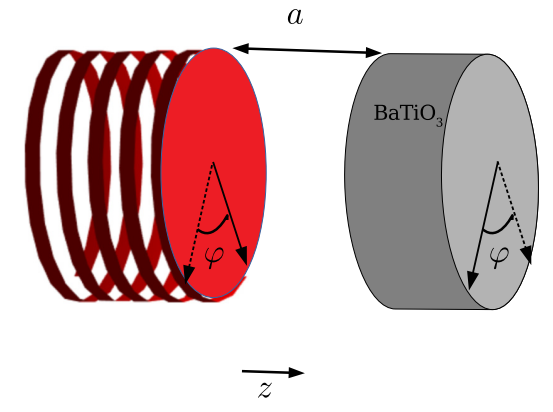

(b)

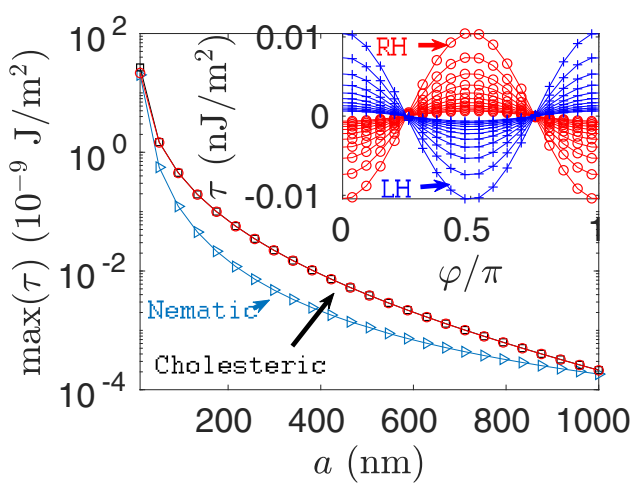

(d)

FIG. 4. The Casimir torque between a birefringent $\left(\mathrm{BaTiO}_{3}\right)$ crystal and a cholesteric liquid crystal. (a) A left-handed crystal facing a birefringent one. (b) A right-handed crystal facing a birefringent one. (c) The Casimir torque as a function of misalignment angle at a distance of $a=10 \mathrm{~nm}$. The different curves are as follows. The triangles pointing right show the usual result for a nematic liquid crystal. Red circles: left-handed crystal, $\delta=0$. Blue triangles pointing left: left-handed crystal, $\delta \neq 0$. Magenta crosses: right-handed crystal, $\delta=0$. Black squares: right-handed crystal, $\delta \neq 0$. (d): The amplitude of the Casimir torque as a function of separation. The triangles pointing right show the nematic case. The cholesteric case is shown by the other two curves, of which the red circles represents $\delta=0$ and the black squares show the perturbed case. The inset of panel (d) shows the Casimir torque for the cases (a) (red circles) and (b) (blue crosses) at distances between 380 and $800 \mathrm{~nm}$.

sample is presently experimentally accessible [29], this could make experimental observation of the torque easier at larger distances. The inset of Fig. 4(d) shows that the angular dependence remains the same at large distances (between 380 and $800 \mathrm{~nm}$ ): the positions of the extrema and the nodes do not change as a function of distance. The higher-order correction of the $\mathrm{BCH}$ formula [Eq. (36)] is negligible in this separation range, contributing significantly only at distances of $\sim 10 \mathrm{~nm}$.

Next, we proceed to the case of two liquid crystals facing each other. Here we distinguish between the so-called homo and heterochiral cases, shown in Figs. 5 and 6, respectively. In the first case two right-handed or two left-handed crystals face each other, and in the second case a left-handed crystal is faced with a right-handed one.

First we will discuss the homochiral case, as shown in Fig. 5. Figures 5(a) and 5(b) illustrate the possible configurations: two right- and two left-handed crystals. Figure 5(c) shows a plot of the Casimir torque as a function of misalignment angle at a distance of $10 \mathrm{~nm}$. For reference, the case of two nematic liquid (light blue triangles) was also included. As in the previous case (see Fig. 4), the leading-order term shifts the usual sine to a cosine. However, unlike the previous case, here the higher-order terms decrease the amplitude of the torque in one direction, which does give rise to a deviation from the sinusoidal form. This short distance is the only range where the finite pitch length effects, represented by the higher-order term in Eq. (36), actually contribute significantly. At this range, it seems as though the torque for the lefthanded configuration is opposite of that of the right-handed one. However, at larger distances it can be seen that this is not the case. In Fig. 5(d) the Casimir torque is plotted as a function of the misalignment angle for distances between 380 and $800 \mathrm{~nm}$. At this distance range, the higher-order term of Eq. (36) contributes negligibly. Still even the lower-order terms give rise to a significant deviation from the sinusoidal behavior from the previous case. Moreover, the position of the extrema changes with the distance, and the nodes are not on the $x$-axis. In other words, the angular dependence changes with the separation distance, which is qualitatively different from the previous case or the case of two birefringent materials. The left- and right-handed configurations do not exhibit opposite torques, but the phase difference between the curves changes as a function of distance as well. It is worth pointing out that, if a particular angle is chosen, near one of the nodes, it becomes possible to see the torque change sign as a function of distance. This kind of sign change has also been reported for biaxial materials [63] and Weyl semi-metals [26], for example.

Next we will address the heterochiral case shown in Fig. 6. Figures 6(a) and 6(b) show the configurations of a left-handed crystal on the left- and a right-handed crystal on the right, and the reverse configuration, respectively. These configura- 


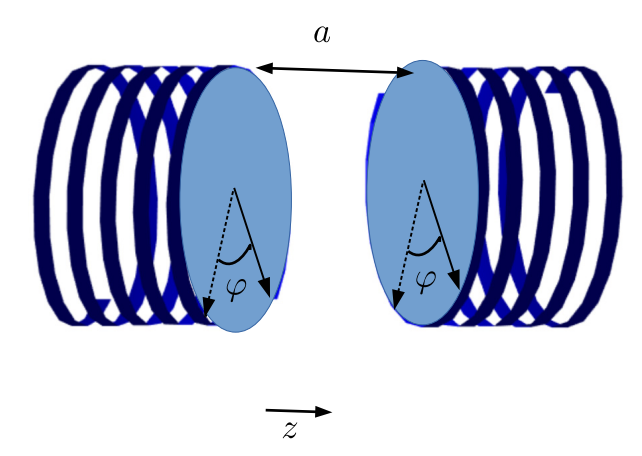

(a)

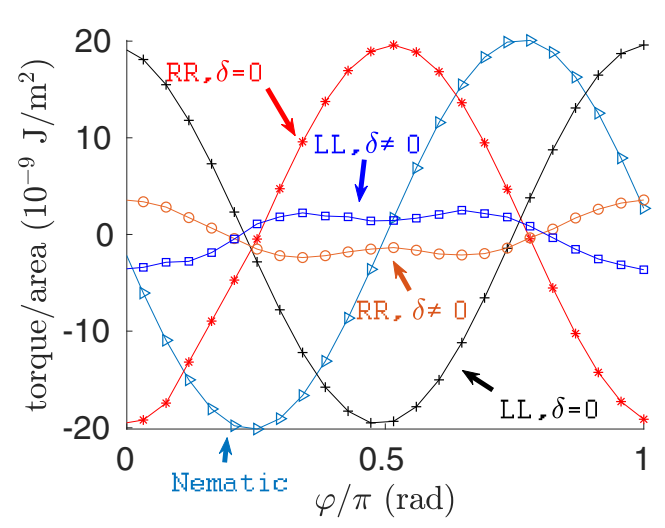

(c)

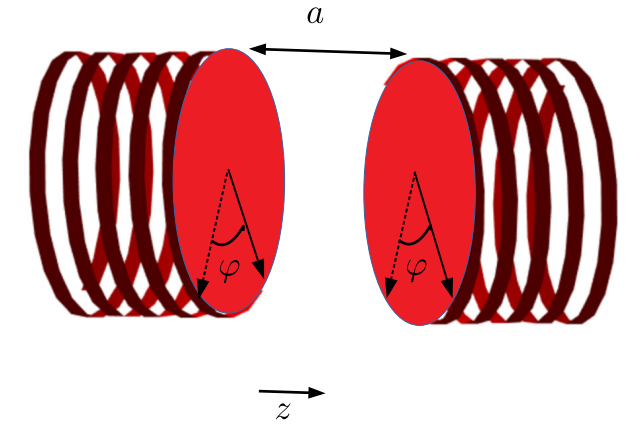

(b)

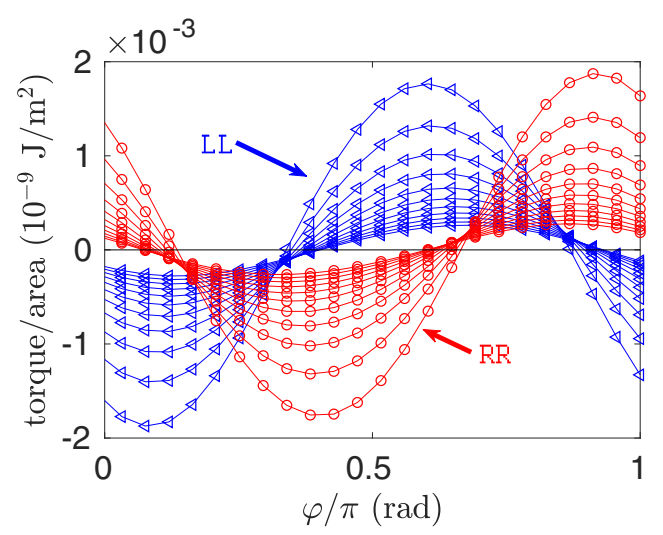

(d)

FIG. 5. The Casimir torque between two cholesteric liquid crystals of identical chirality. (a) Homochiral configuration of two left-handed crystals (b) Homochiral configuration of two right-handed crystals. (c) Casimir torque as a function of misalignment angle at a distance of $a=10 \mathrm{~nm}$. The different curves are as follows. Triangles pointing right: two nematic liquid crystals. Black crosses: unperturbed, case (a). Blue squares: perturbative contribution to case (a). Red circles: unperturbed case (b). Orange circles: perturbative contribution to case (b). (d) The Casimir torque as a function of misalignment angle for distances between 380 an $800 \mathrm{~nm}$. The blue triangles represent case (a) and the red circles represent case (b).

tions are expected to be physically indistinguishable. To test this, we plot the different contributions to the torque as a function of misalignment angle again at $10-\mathrm{nm}$ distance, see Fig. 6(c). It can be seen that the difference between the torque of Figs. 6(a) and 6(b) is nonnegligible if the higher-order term in Eq. (36) is omitted [see the black crosses in Fig. 6(c)]. However, the inclusion of the higher-order terms renders this difference negligible [the red asterisks in Fig. 6(c)]. So even though the higher-order corrections themselves are different [as indicated by the orange circles and blue squares in Fig. 6(c)], the total torque is identical for both configurations. Finally, the torque is plotted as a function of the misalignment angle for distances of 380 to $800 \mathrm{~nm}$ in Fig. 6(d). Here the torque behaves qualitatively similar to the previous case, with the phase somewhere in between that of the torque for the two homochiral configurations of Fig. 5(d). As expected, the torques for the configurations of Figs. 6(a) and 6(b) are identical. Since the lowest-order term in the $\mathrm{BCH}$ expansion assumes pitch length is infinite, it can lead to unphysical results as shown in Fig. 6(c). A more realistic inclusion of finite pitch length effects is needed, in this case in the form of the higher-order correction to the $\mathrm{BCH}$ formula. In all considered cases, these corrections are most significant at short distances of about $10 \mathrm{~nm}$. This is most likely because the end of the pitch is relatively close to the other surface at this range. This way, the pitch end can be "felt" by the other surface, and affect the Casimir-Lifshitz torque.

\section{CONCLUSION AND OUTLOOK}

To understand the effects of cholesteric chirality on the Casimir-Lifshitz torque, we modeled a cholesteric liquid crystal as a uniaxial planar multilayer system. The system consists of identical layers of equal thicknesses, but the orientation of each layer differs slightly from that of the adjacent one. This model allows us to derive an analytical simplification of the Fresnel matrices as an expansion in this small orientation difference, by means of the Baker-Campbell-Hausdorff $(\mathrm{BCH})$ formula.

Numerically, we obtained results that are appreciably different from the case of two semi-infinite birefringent plates, which is a valid approximation for nematic liquid crystals [29,32]. In particular, in the case of a birefringent half space facing a cholesteric liquid crystal, the leading-order term in the $\mathrm{BCH}$ expansion shifts the angular dependence of the torque by about $\pm \pi / 2$ radians, depending on the chirality of the liquid crystal. At very short distances of about $10 \mathrm{~nm}$, the higher-order $\mathrm{BCH}$ term gives rise to a significant deviation from the usual sinusoidal shape of the torque as a function of misalignment angle. Moreover, the amplitude of the torque 


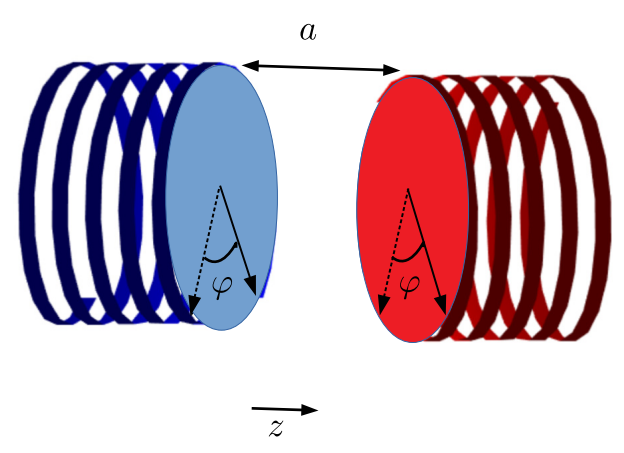

(a)

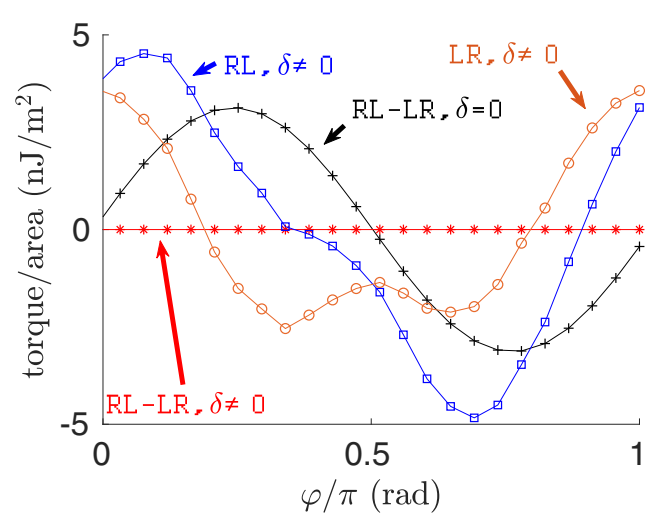

(c)

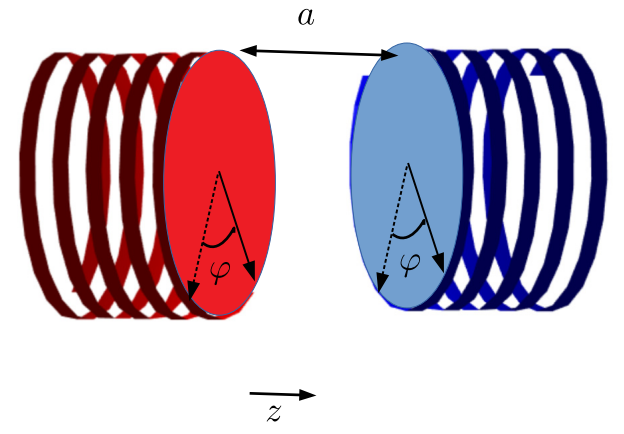

(b)

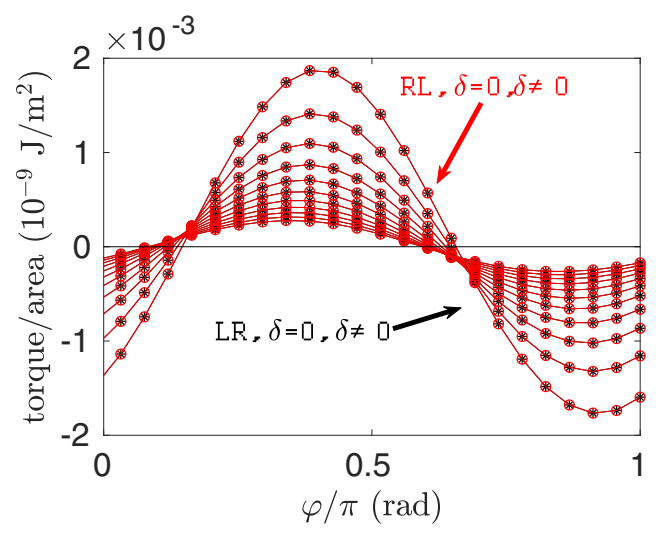

(d)

FIG. 6. The Casimir torque for the heterochiral case. (a) The left-handed crystal is placed on the left, and the right-handed crystal is on the right. (b) The reverse of panel (a). (c) Several different contributions at 10-nm distance. Black crosses: difference between case (a) and case (b), including only lowest-order terms. Red asterisks: idem, including higher-order terms. Orange circles: perturbative contribution to case (a), blue squares: perturbative contribution to case (b). (d) Casimir torque at distances between 380 and $800 \mathrm{~nm}$, both for case (a) (black asterisks), and case (b) (red circles).

decreases more slowly as a function of distance than in the nematic case. Since the configuration of a birefringent crystal faced with a liquid crystal is presently experimentally accessible [29], we believe that especially this later result can be useful for detecting the torque at large separation distances.

The case of two cholesteric liquid crystals consists of three physically different configurations: two homochiral ones (two left-handed crystals or two right-handed crystals), and one heterochiral one. In each case, the higher-order term of the $\mathrm{BCH}$ expansion, which is associated with finite pitch length, contributes significantly at about 10-nm distance. We showed that it must be included to obtain physically consistent results in the heterochiral case. At larger distances, the angular dependence of the torque turns out to change as a function of distance, while again significantly deviating from the known sinusoidal behavior. Moreover, for certain values of the misalignment angle, the torque can change sign as a function of distance. Present technology does not yet make it possible to reach a sufficient degree of parallelism to measure the Casimir torque between two liquid crystals with satisfactory accuracy [13]. However, present efforts directed at a resolution of this problem [7] give us hope that it may be possible in the future to measure the torque for such a case.

Possible future endeavors include calculations beyond the approximations used here. For example, one could allow discontinuity in the inhomogeneous dielectric function. This would make it possible to study the effect of a thin water layer between two layers of the cholesteric liquid crystal. Alternatively, one could explore a larger distance range in which case one needs to investigate the effects of a finite total thickness. Finally, the approximate solution of the Maxwell equations obtained here may be of interest for the study of cholesterics in different contexts, or for the understanding of chiral media in general.

\section{ACKNOWLEDGMENTS}

We acknowledge funding from the Key Project No. 12034019 of the National Natural Science Foundation of China (WB and RP) and the Singapore Ministry of Education Academic Research Fund Tier 1 Grant No. RG160/19(S) (B-SL).
[1] H. B. G. Casimir, On the attraction between two perfectly conducting plates, Indag. Math. 10, 261 (1948).

[2] E. M. Lifshitz, The theory of molecular attractive forces between solids, Sov. Phys. JETP 29, 94 (1956); I. E.
Dzyaloshinskii, E. M. Lifshitz, and L. P. Pitaevskii, The general theory of van der Waals forces, Adv. Phys. 10, 165 (1961).

[3] V. Adrian Parsegian, Van der Waals Forces (Cambridge University Press, Cambridge, England, 2006). 
[4] M. Bordag, G. L. Klimchitskaya, U. Mohideen, and V. M. Mostepanenko, Advances in the Casimir Effect (Oxford University Press, Oxford, 2009).

[5] S. Y. Buhmann, Dispersion Forces I: Macroscopic Quantum Electrodynamics and Ground-State Casimir, Casimir-Polder and van der Waals Forces, 1st ed., Springer Tracts in Modern Physics 247 (Springer-Verlag, Berlin, 2012).

[6] L. M. Woods, D. A. R. Dalvit, A. Tkatchenko, P. RodriguezLopez, A. W. Rodriguez, and R. Podgornik, Materials perspective on Casimir and van der Waals interactions, Rev. Mod. Phys. 88, 045003 (2016).

[7] R. I. P. Sedmik, Casimir and non-Newtonian force experiment (CANNEX): Review, status, and outlook, Int. J. Mod. Phys. A 35, 02 n03 (2020).

[8] F. M. Serry, D. Walliser, and G. J. Maclay, The anharmonic Casimir oscillator - the Casimir effect in a model microelectromechanical system, J. Microelec. Sys. 4, 193 (1995).

[9] W. Broer, G. Palasantzas, J. Knoester, and V. B. Svetovoy, Significance of the Casimir force and surface roughness for actuation dynamics of MEMS, Phys. Rev. B 87, 125413 (2013).

[10] W. Broer, H. Waalkens, V. B. Svetovoy, J. Knoester, and G. Palasantzas, Nonlinear Actuation Dynamics of Driven Casimir Oscillators with Rough Surfaces, Phys. Rev. Applied 4, 054016 (2015).

[11] M. Sedighi, W. H. Broer, S. van der Veeke, V. B. Svetovoy, and G. Palasantzas, Influence of materials' optical response on actuation dynamics by Casimir forces, J. Phys.: Condens. Matter 27, 214014 (2015).

[12] F. Tajik, M. Sedighi, A. A. Masoudi, H. Waalkens, and G. Palasantzas, Dependence of chaotic behavior on optical properties and electrostatic effects in double-beam torsional Casimir actuation, Phys. Rev. E 98, 022210 (2018).

[13] T. Gong, M. R. Corrado, A. R. Mahbub, C. Shelden, and J. N. Munday, Recent progress in engineering the Casimir effectapplications to nanophotonics, nanomechanics, and chemistry, Nanophotonics 10, 523 (2021).

[14] J. N. Israelachvili, Intermolecular and Surface Forces (Elsevier, Amsterdam, 2011).

[15] J. C. Hopkins, R. Podgornik, W.-Y. Ching, R. H. French, and V. A. Parsegian, Disentangling the effects of shape and dielectric response in van der Waals interactions between anisotropic bodies, J. Phys. Chem. C 119, 19083 (2015).

[16] V. A. Parsegian and G. H. Weiss, Dielectric anisotropy and the van der Waals interaction between bulk media, J. Adhesion 3, 259 (1972).

[17] Y. S. Barash and V. L. Ginzburg, Electromagnetic fluctuations in matter and molecular (van der Waals) forces between them, Sov. Phys. Usp. 18, 305 (1975).

[18] Y. S. Barash, Moment of van der Waals forces between anisotropic bodies, Radiophysics Quantum Electronics 21, 1138 (1978).

[19] D. W. Berreman, Optics in stratified and anisotropic media: 4×4-matrix formulation, J. Opt. Soc. Am. 62, 502 (1972).

[20] P. Yeh, Optics of anisotropic layered media: A new $4 \times 4$ matrix algebra, Surf. Sci. 96, 41 (1980).

[21] J. Lekner, Reflection and refraction by uniaxial crystals, J. Phys.: Condens. Matter 3, 6121 (1991).

[22] C.-G. Shao, A.-H. Tong, and J. Luo, Casimir torque between two birefringent plates, Phys. Rev. A 72, 022102 (2005).
[23] T. G. Philbin and U. Leonhardt, Alternative calculation of the Casimir forces between birefringent plates, Phys. Rev. A 78, 042107 (2008).

[24] W. Broer, J. Y. H. Liow, and B.-S. Lu, Maxwell eigenmode approach to the Casimir-Lifshitz torque, Phys. Rev. A 100, 012514 (2019).

[25] N. Emelianova, I. V. Fialkovsky, and N. Khusnutdinov, Casimir effect for biaxial anisotropic plates with surface conductivity, Mod. Phys. Lett. A 35, 2040012 (2020).

[26] L. Chen and K. Chang, Chiral-anomaly-driven Casimir-Lifshitz Torque between Weyl semimetals, Phys. Rev. Lett. 125, 047402 (2020).

[27] M. B. Farias, A. A. Zyuzin, and T. L. Schmidt, Casimir force between Weyl semimetals in a chiral medium, Phys. Rev. B 101, 235446 (2020).

[28] R. Zeng, C. Wang, X. Zeng, H. Li, S. Yang, Q. Li, and Y. Yang, Casimir torque and force in anisotropic saturated ferrite threelayer structure, Opt. Express 28, 7425 (2020).

[29] D. A. T. Somers, J. L. Garrett, K. J. Palm, and J. N. Munday, Measurement of the Casimir torque, Nature (London) 564, 386 (2018).

[30] F. K. P. Haddadan, A. Naji, A. K. Seifi, and R. Podgornik, Pseudo-Casimir interactions across nematic films with disordered anchoring axis, J. Phys.: Condens. Matter 26, 075103 (2014).

[31] F. Karimi Pour Haddadan, A. Naji, and R. Podgornik, Casimirlike interactions and surface anchoring duality in bookshelf geometry of smectic-a liquid crystals, Soft Matter 15, 2216 (2019).

[32] D. A. T. Somers and J. N. Munday, Rotation of a liquid crystal by the Casimir torque, Phys. Rev. A 91, 032520 (2015).

[33] P. E. Kornilovitch, Van der Waals interaction in uniaxial anisotropic media, J. Phys.: Condens. Matter 25, 035102 (2012); Corrigendum: Van der Waals interaction in uniaxial anisotropic media (2013 J. Phys.: Condens. Matter 25 035102), 30, 189501 (2018)

[34] A. Ryabchun and A. Bobrovsky, Cholesteric liquid crystal materials for tunable diffractive optics, Adv. Opt. Mater. 6, 1800335 (2018).

[35] G. Veble and R. Podgornik, Dispersion interactions in stratified anisotropic and optically active media at all separations, Phys. Rev. B 80, 075422 (2009).

[36] R. Podgornik and V. A. Parsegian, Van der Waals interactions in a dielectric with continuously varying dielectric function, J. Chem. Phys. 121, 7467 (2004).

[37] B.-S. Lu and R. Podgornik, Van der Waals torque and force between dielectrically anisotropic layered media, J. Chem. Phys. 145, 044707 (2016).

[38] B.-S. Lu, van der Waals torque and force between anisotropic topological insulator slabs, Phys. Rev. B 97, 045427 (2018).

[39] N. J. Higham, Functions of Matrices: Theory and Computation (Society for Industrial and Applied Mathematics, Philadelphia, PA, 2008), pp. $x x+425$.

[40] P. Barcellona, H. Safari, A. Salam, and S. Y. Buhmann, Enhanced Chiral Discriminatory Van Der Waals Interactions Mediated by Chiral Surfaces, Phys. Rev. Lett. 118, 193401 (2017).

[41] Q.-D. Jiang and F. Wilczek, Chiral Casimir forces: Repulsive, enhanced, tunable, Phys. Rev. B 99, 125403 (2019). 
[42] H. Safari, P. Barcellona, S. Y. Buhmann, and A. Salam, Medium-assisted van der Waals dispersion interactions involving chiral molecules, New J. Phys. 22, 053049 (2020).

[43] N. C. Passler and A. Paarmann, Generalized 4 x 4 matrix formalism for light propagation in anisotropic stratified media: study of surface phonon polaritons in polar dielectric heterostructures, J. Opt. Soc. Am. B 34, 2128 (2017).

[44] F. S. S. Rosa, D. A. R. Dalvit, and P. W. Milonni, CasimirLifshitz theory and metamaterials, Phys. Rev. Lett. 100, 183602 (2008).

[45] See Supplemental Material at http://link.aps.org/supplemental/ 10.1103/PhysRevResearch.3.033238 for detailed derivations, explicit formulas, and comparison to earlier results.

[46] J. Schwinger, L. L. DeRaad, and K. A. Milton, Casimir effect in dielectrics, Ann. Phys. (NY) 115, 1 (1978).

[47] A. Lambrecht, P. A. M. Neto, and S. Reynaud, The Casimir effect within scattering theory, New J. Phys. 8, 243 (2006).

[48] S. Scheel and S. Y. Buhmann, Macroscopic quantum electrodynamics - concepts and applications, Acta Physica Slovaca 58, 675 (2008).

[49] N. G. Van Kampen, B. R. A. Nijboer, and K. Schram, On the macroscopic theory of Van der Waals forces, Phys. Lett. A 26, 307 (1968)

[50] K. Schram, On the macroscopic theory of retarded van der Waals forces, Phys. Lett. A 43, 282 (1973).

[51] Y. S. Barash and V. L. Ginzburg, Some problems in the theory of van der Waals forces, Soviet Phys. Usp. 27, 467 (1984).

[52] F. Intravaia and R. Behunin, Casimir effect as a sum over modes in dissipative systems, Phys. Rev. A 86, 062517 (2012).

[53] B. Fornberg, Generation of finite difference formulas on arbitrarily spaced grids, Math. Comput. 51, 699 (1988).

[54] M. Abramowitz and I. A. Stegun, Handbook of Mathematical Functions with Formulas, Graphs, and Mathematical Tables, 9th Dover printing, 10th GPO printing ed. (Dover, New York, 1964).

[55] The matrices may still be approximately jointly diagonalizable. However, at this point it is not clear how to estimate this approximation, or what the common eigenvector basis should be.

[56] B.-S. Lu, The Casimir effect in topological matter, Universe 7, 237 (2021).

[57] G. Kocakülah, O. Köysal, and A. Kahyaoğlu, Electro-optical performance investigation of cholesteric liquid crystal containing azo dye: Light shutter device application, J. Electron. Mater. 50, 497 (2021).

[58] P. J. van Zwol, G. Palasantzas, and J. T. M. De Hosson, Influence of random roughness on the Casimir force at small separations, Phys. Rev. B 77, 075412 (2008).

[59] P. J. van Zwol, V. B. Svetovoy, and G. Palasantzas, Distance upon contact: Determination from roughness profile, Phys. Rev. B 80, 235401 (2009).

[60] W. Broer, G. Palasantzas, J. Knoester, and V. B. Svetovoy, Roughness correction to the Casimir force beyond perturbation theory, Europhys. Lett. 95, 30001 (2011).

[61] W. Broer, G. Palasantzas, J. Knoester, and V. B. Svetovoy, Roughness correction to the Casimir force at short separations: Contact distance and extreme value statistics, Phys. Rev. B 85 , 155410 (2012).

[62] J. N. Munday, D. Iannuzzi, Y. Barash, and F. Capasso, Torque on birefringent plates induced by quantum fluctuations, Phys. Rev. A 71, 042102 (2005).

[63] P. Thiyam, P. Parashar, K. V. Shajesh, O. I. Malyi, M. Boström, K. A. Milton, I. Brevik, and C. Persson, Distance-Dependent Sign Reversal in the Casimir-Lifshitz Torque, Phys. Rev. Lett. 120, 131601 (2018).

[64] S. Wu, A semiempirical model for liquid-crystal refractive index dispersions, J. Appl. Phys. 69, 2080 (1991).

[65] M. R. de la Fuente and D. Dunmur, Dielectric Properties of Liquid Crystals, in Handbook of Liquid Crystals (Wiley, New York, 2014), Chap. 4, pp. 1-46.

[66] S.-T. Wu, C. Wu, M. Warenghem, and M. Ismaili, Refractive index dispersions of liquid crystals, Opt. Eng. 32, 1775 (1993).

[67] L. Bergström, Hamaker constants of inorganic materials, Adv. Colloid Interface Sci. 70, 125 (1997). 